\title{
S3-Leitlinie "Chirurgie der Adipositas»: Kommentierte Kurzfassung
}

\author{
Norbert Runkel $^{\mathrm{a}} \quad$ Mario Colombo-Benkmann $^{\mathrm{b}} \quad$ Thomas P. Hüttl $^{\mathrm{c}} \quad$ Harald Tigges $^{\mathrm{d}} \quad$ Oliver Mann $^{\mathrm{e}}$ \\ ${ }^{a}$ Klinik für Allgemein-, Visceral- und Kinderchirurgie, Kliniken Villingen, Villingen-Schwenningen,

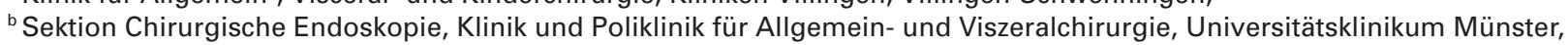 \\ ${ }^{c}$ Adipositas Zentrum München, \\ ${ }^{d}$ Adipositas Zentrum Bodensee und Viszeral-, Thorax- und Gefäßchirurgie, Klinikum Konstanz, \\ ${ }^{e}$ Klinik und Poliklinik für Allgemein-, Visceral- und Thoraxchirurgie, Universitätsklinikum Hamburg-Eppendorf, Hamburg, Deutschland
}

Schlüsselwörter

Adipositas - Adipositastherapie - Adipositaschirurgie ·

Bariatrische Chirurgie

\section{Zusammenfassung}

Hintergrund: Übergewicht und Adipositas haben in Deutschland endemische Ausmaße erreicht. Infolgedessen nimmt die Zahl der Adipositas-Operationen (bariatrische Chirurgie) rasant zu. Neue minimal invasive Operationsmethoden und die Zunahme wissenschaftlicher Evidenz tragen zur Erkenntnis bei, dass im multimodalen Behandlungskonzept der Adipositas und ihrer Folgeerkrankungen chirurgische Konzepte integriert werden müssen. Methoden: Systematische Literaturrecherche, Evidenzklassifikation, Entwicklung von graduierten Empfehlungen und interdisziplinäre Konsentierung. Ergebnisse: Adipositas-Operationen sind evidenzbasierte Behandlungsoptionen, die eine multidisziplinäre Evaluation und Vorbereitung, kompetente Durchführung und langfristige Nachsorge benötigen. Die Leitlinie bestätigt das traditionelle, BMIbasierte Indikationsspektrum (BMI $>40 \mathrm{~kg} / \mathrm{m}^{2}$ oder $>35 \mathrm{~kg} / \mathrm{m}^{2}$ mit Sekundärerkrankungen) und erweitert es durch Wegfall von Altersbegrenzungen und vielen Kontraindikationen. Voraussetzung zur Operation ist die Erschöpfung einer strukturierten konservativen Therapie über 6-12 Monate oder die Aussichtslosigkeit derselben. Diabetes mellitus Typ 2 wird als potenzielles Indikationskriterium bei einem BMI $<35 \mathrm{~kg} / \mathrm{m}^{2}$ aufgenommen (metabolische Chirurgie). Die Verfahrenswahl ist individualisiert; Standardtechniken sind Magenband, Magen-Bypass, Schlauchmagen und biliopankreatische Diversion. Um spezifischen Mangelerscheinungen vorzubeugen, und weil die Adipositas als chronische Erkrankung verstanden werden sollte, muss auch nach Operationen die Nachsorge strukturiert und langfristig geplant werden. Schlussfolgerung: Die S3-Leitlinie beinhaltet evidenzbasierte Empfehlungen zur Indikation, Verfahrenswahl, Technik und Nachsorge. Die Implementierung der Leitlinie in Praxis und Klinik und deren Beachtung durch Kostenträger/MDK wird eine Optimierung der Patientenversorgung zur Folge haben.
Keywords

Obesity · Obesity treatment - Obesity surgery . Bariatric surgery

\section{Summary}

Evidence-Based Guidelines for Bariatric Surgery: Commented Short Version

Background: Overweight and obesity have reached endemic dimensions in Germany. Consequently, the number of obesity procedures (bariatric surgery) is rising rapidly. These guidelines outline the implication of the increasing scientific evidence for bariatric surgery and its role within a multimodal treatment concept. Methods: Systematic literature search, classification of evidence, structured development of recommendation and interdisciplinary consensus. Results: Obesity operations are evidence-based treatment options that require a multidisciplinary evaluation and preparation, competent performance and long-term follow-up. The guideline extends the traditional BMI-based spectrum of indications (BMI $>40 \mathrm{~kg} / \mathrm{m}^{2}$ or $>35 \mathrm{~kg} / \mathrm{m}^{2}$ with associated complications) through the elimination of age restrictions and many contraindications. Surgery requires the exhaustion of a structured conservative therapy for a minimum of 6-12 months or the assumption of its failure. Diabetes mellitus type 2 is a potential indication criterion in patients with $\mathrm{BMI}<35 \mathrm{~kg} / \mathrm{m}^{2}$ (metabolic surgery). The selection of patients for surgery is an individualized process. Standard techniques are laparoscopic gastric banding, gastric bypass, sleeve gastrectomy and biliopancreatic diversion. Postoperative follow-up care should be structured to prevent specific metabolic deficiencies and should be life-long, recognizing the chronicity of obesity. Conclusion: The guideline contains evidence-based recommendations for the indication of bariatric surgery, choice of procedure, technical aspects and follow-up. The implementation of the guideline in clinical practice and its recognition by health care providers will contribute to improved care of obese patients.

\section{KARGER}

Fax +497614520714

Information@Karger.de

www.karger.com (c) 2011 S. Karger GmbH, Freiburg

$1662-6664 / 11 / 0276-0465 \$ 38.00 / 0$

Accessible online at:

www.karger.com/vim
Prof. Dr. Dr. h.c. Norbert Runkel

Klinik für Allgemein-, Visceral- und Kinderchirurgie

Kliniken Villingen

Vöhrenbacherstraße 15, 78050 Villingen-Schwenningen, Deutschland

Tel. +49 7721 93-3301, Fax -3399

avc@sbk-vs.de 


\section{Einleitung}

Das Körpergewicht der Deutschen ist in den letzten Jahrzehnten kontinuierlich angestiegen. Jeder Zweite ist übergewichtig und jeder Fünfte ist adipös. Die Klassifikation der Adipositas beruht auf dem Body Mass Index (BMI), der sich aus Körpergewicht dividiert durch Körpergröße im Quadrat $\left(\mathrm{kg} / \mathrm{m}^{2}\right)$ errechnet. Für Europäer teilt man die Adipositas in Grad I (BMI 30-34,9 kg/m²), Grad II (BMI 35-39,9 kg/m²) und Grad III $\left(B M I \geq 40 \mathrm{~kg} / \mathrm{m}^{2}\right)$ ein. Die Adipositas wird üblicherweise bei einem BMI $\geq 40 \mathrm{~kg} / \mathrm{m}^{2}$ oder BMI $\geq 35 \mathrm{~kg} / \mathrm{m}^{2}$ mit Komorbidität als krankhaft (morbid) bezeichnet. Häufig findet man im Schrifttum die Ausdrücke super- oder megaadipös bei Patienten ab einem BMI $>50 \mathrm{~kg} / \mathrm{m}^{2}$.

Adipositas ist mit einer Reihe bedeutsamer Erkrankungen assoziiert, wie z.B. Insulinresistenz, Diabetes mellitus Typ 2, Lipidstoffwechselstörungen, Bluthochdruck (metabolisches Syndrom), Gallensteinen, bestimmten Krebsarten, gastroösophagealer Refluxkrankheit (GERD), Steatosis hepatis, degenerativen Gelenkerkrankungen, obstruktivem Schlaf-ApnoeSyndrom, Hypoventilationssyndrom und psychiatrischen Erkrankungen. Die morbide Adipositas reduziert die Lebenserwartung um bis zu 20 Jahre [1].

In umfangreichen Metaanalysen [2-4] wurde die Wirksamkeit von Adipositas-Operationen (bariatrische Chirurgie) hinsichtlich substanzieller Gewichtsreduktion und Rückbildung von Sekundärfolgen nachgewiesen (Evidenzlevel (EL) 1a). In der prospektiven kontrollierten «Swedish Obesity Subjects (SOS)»-Studie [5] wurden bariatrische Eingriffe $(\mathrm{n}=641)$ mit konservativen Standardbehandlungen $(\mathrm{n}=627)$ verglichen (vertikale Gastroplastik $(\mathrm{n}=451)$, Magenband $(\mathrm{n}=156)$, Magen-Bypass $(\mathrm{n}=34))($ EL 2b). In der konservativen Gruppe lag das Körpergewicht nach 10 Jahren 1,6\% über dem Ausgangsgewicht sowie in der operativen Gruppe nach 2 Jahren 23\% und nach 10 Jahren 16\% darunter (Magenband 14\%, vertikale Gastroplastik 16\%, Magen-Bypass 25\%). Die Langzeitergebnisse blieben in der SOS-Studie über 15 Jahre stabil (EL 2b) [6]. Durch den Rückgang von assoziierten Erkrankungen, insbesondere durch Reduktion von Myokardinfarkten, Diabetes mellitus und Krebs, kann die Langzeitletalität um bis zu $40 \%$ verringert werden (EL 2b) [6, 7].

Auch in Deutschland stiegen die Operationszahlen in den letzten Jahren an. Beigetragen haben dazu die Entwicklung neuer Techniken, die Minimierung der operativen Zugangswege, eine enorme Zunahme wissenschaftlicher Studien und die Erkenntnis, dass eine dauerhafte Gewichtsreduktion mit Rückbildung der Sekundärerkrankungen häufig nur durch eine Operation erzielt werden kann.

Das Ziel der Leitlinie ist die systematische Auswertung der wissenschaftlichen Evidenz, um Ärzten, mitbetreuenden Berufsgruppen und Patienten Entscheidungshilfen zu geben. Die Leitlinie soll dadurch ein Instrument zur Qualitätsoptimierung der Adipositasbehandlung darstellen. In diesem Artikel werden die Empfehlungen mit einem kommentierenden Begleittext wiedergegeben. Die Originalversion mit detaillierten Informationen zur Methodik, dem Evidenz-Text und den Literaturzitaten ist über die Internetseiten der Arbeitsgemeinschaft der Wissenschaftlichen Medizinischen Fachgesellschaften e.V. (AWMF; www.awmf.de) oder der an der Leitlinie beteiligten medizinischen Fachgesellschaften abrufbar.

\section{Methodik}

Die S3-Leitlinie erfüllt die Anforderungen der systematischen Entwicklung und interdisziplinären Konsentierung («Deutsches Leitlinien-Bewertungsinstrument» der AWMF und des Ärztlichen Zentrums für Qualität in der Medizin (ÄZQ), 2005; www.delbi.de). Als Grundlage diente die evidenzbasierte Leitlinie der European Association for Endoscopic Surgery (EAES) unter der Federführung des hier ebenfalls mitwirkenden S. Sauerland aus dem Jahre 2005 [8]. Die Graduierung in EL und Empfehlungsgrade, die mit den Worten «soll», «sollte», «kann» und entsprechenden Pfeilsymbolen beschrieben wurden, erfolgte nach den Vorschlägen des Oxford Centre for Evidence-Based Medicine. In der Regel bestimmte die Qualität der Evidenz (Evidenzstärke) den Empfehlungsgrad (Tab. 1). Alle Empfehlungen wurden auf zwei formalen Konsensus-Konferenzen im «starken Konsens»
Tab. 1. Graduierung der Evidenz- und Empfehlungsstärke

\begin{tabular}{|c|c|c|c|c|}
\hline Studienqualität & Evidenzstärke & Empfehlung & Beschreibung & Symbol \\
\hline $\begin{array}{l}\text { Systematische Übersichtsarbeit (Meta- } \\
\text { Analyse) oder RCT (Therapie) oder } \\
\text { Kohortenstudien (Risikofaktoren, } \\
\text { Diagnostik); von hoher Qualität }\end{array}$ & hoch & «soll» & $\begin{array}{l}\text { starke } \\
\text { Empfehlung }\end{array}$ & $\Uparrow \Uparrow$ \\
\hline $\begin{array}{l}\text { RCT oder Kohortenstudien von } \\
\text { eingeschränkter Qualität }\end{array}$ & mäßig & «sollte» & Empfehlung & $\Uparrow$ \\
\hline $\begin{array}{l}\text { RCT oder Kohortenstudien von } \\
\text { schlechter Qualität, alle anderen } \\
\text { Studiendesigns, Expertenmeinung }\end{array}$ & schwach & «kann» & $\begin{array}{l}\text { Empfehlung } \\
\text { offen }\end{array}$ & $\Leftrightarrow$ \\
\hline
\end{tabular}


(Zustimmung von $>95 \%$ der Teilnehmer) verabschiedet. Das Manuskript wurde den beteiligten Fachgesellschaften zur Kommentierung vorgelegt und dann von der Expertengruppe verabschiedet.

\section{Empfehlungen}

\section{Evaluation und Diagnostik}

- Eine operative Maßnahme zur Gewichtsreduktion erfordert eine vorhergehende interdisziplinäre Stellungnahme. $\Uparrow \Uparrow$

- In diesem Zusammenhang sollte eine Vorstellung bei einem in der konservativen Adipositastherapie erfahrenen Arzt erfolgen. $\Uparrow$

- Bereits präoperativ sollen die Patienten von einer Ernährungsfachkraft mitbetreut werden. $\Uparrow \Uparrow$

- In Abhängigkeit von der Komorbidität des adipösen Patienten sollen weitere Fachdisziplinen und Experten hinzugezogen werden. $\Uparrow \Uparrow$

- Insbesondere sollte bei allen Patienten die Konsultation eines möglichst in der Therapie der Adipositas tätigen klinischen Psychologen, Psychosomatikers oder Psychiaters in Betracht gezogen werden. $\Uparrow$

- Große Oberbaucheingriffe wie die bariatrischen Operationen bedürfen einer umfassenden Vorbereitung mit gründlicher Anamneseerhebung unter Erfassung und Dokumentation aller Begleiterkrankungen, der aktuellen Medikation, der Beschwerden und der Symptome. $\Uparrow \Uparrow$

- Eine Ösophagogastroduodenoskopie soll im Vorfeld bariatrischer Operationen durchgeführt werden. $\Uparrow \Uparrow$

- Ursachen der Adipositas sind vor einer bariatrischen Maßnahme auszuschließen. Generell trifft das für die Hypothyreose zu, bei klinischen Anzeichen auch für andere. $\Uparrow \Uparrow$

\section{Kommentar}

Die präoperative Evaluation erfolgt nach den gängigen Standards für große Oberbaucheingriffe. Dazu gehören die Differentialabklärung und eine Ösophagogastroduodenoskopie. Einbezogen werden sollen ein mit der konservativen Therapie der Adipositas vertrauter Spezialist (z.B. Ernährungsmediziner) und eine Ernährungsfachkraft (Diätassistent oder Ökotrophologe mit inhaltlich dem Diätassistentengesetz gleichwertiger Ausbildung). Andere Fachrichtungen werden bei Bedarf konsultiert. Wichtig ist die präoperative Erkennung obstruktiver Lungenfunktionsstörungen, die mit steigendem BMI sehr häufig auftreten (EL 2b) [9]. Ein SchlafApnoe-Syndrom ist bei 40-91\% der adipösen Patienten nachweisbar (EL 4) [10, 11].

Die Konsultation von klinischen Psychologen, Psychiatern oder Psychosomatikern ist zwar nicht regelhaft, aber doch häufig notwendig, denn die Prävalenz affektiver, Angst-, Ess- und Persönlichkeitsstörungen nimmt mit steigendem BMI zu (EL 2b) $[12,13]$. Empfehlungen über die Inhalte einer Evaluation des psychischen Zustands liegen vor (EL 4) [14], aber es ist unklar, ob ein psychologisches Screening durch Nicht-Spezialisten mittels selbst entworfener Fragebögen zielführend ist. Die psychische Komorbidität hat mit Ausnahme weniger Krankheitsbilder, wie z.B. Bulimia nervosa, weder einen entscheidenden Einfluss auf die Indikationsstellung (EL 2a) [15] noch kann sie als Prädiktor für den postoperativen Gewichtsverlust herhalten (EL 4) [16]. Die Ziele der präoperativen Evaluation sind die ausführliche psychische und biografische Anamnese, die Abklärung der Motivation und des Wissens über den geplanten Eingriff sowie die Erwartungshaltung des Patienten (z.B. Problemlöser, «quick fix») (EL 4) [14, 17].

Eine gezielte präoperative Gewichtsreduktion kann nicht empfohlen werden, weil die Langzeitergebnisse nicht verbessert werden (EL 1b) [18], auch wenn die operative Übersicht im Abdomen durch das verringerte Lebervolumen optimiert werden kann (EL 2b) [19].

\section{Indikation}

- Bei Patienten mit einem BMI $\geq 40 \mathrm{~kg} / \mathrm{m}^{2}$ ohne Kontraindikationen ist bei Erschöpfung der konservativen Therapie nach umfassender Aufklärung eine bariatrische Operation indiziert. $\Uparrow \Uparrow$

- Bei Patienten mit einem BMI zwischen 35 und 40 kg/m² und mit einer oder mehreren Adipositas-assoziierten Folge-/Begleiterkrankungen (z.B. Diabetes mellitus Typ 2, koronare Herzkrankheit usw.) ist ebenfalls eine chirurgische Therapie indiziert, sofern die konservative Therapie erschöpft ist. $\Uparrow \Uparrow$

- Bei Patienten mit einem Diabetes mellitus Typ 2 kann bereits bei einem BMI zwischen 30 und $35 \mathrm{~kg} / \mathrm{m}^{2}$ eine bariatrische Operation im Rahmen einer wissenschaftlichen Studie erwogen werden. $\Leftrightarrow$

- Auch bei einem höheren Lebensalter (>65 Jahre) kann bei gutem Allgemeinzustand eine bariatrische Operation durchgeführt werden, denn das Alter allein stellt keine Kontraindikation dar. $\Uparrow$

- Bei Patienten im höheren Lebensalter soll die Indikation zur bariatrischen Operation besonders begründet werden. Ziel des Eingriffs ist oft die Verhinderung von Immobilität und Pflegebedürftigkeit. $\Uparrow$

- Kinderwunsch stellt keine Kontraindikation zur bariatrischen Chirurgie dar. $\Uparrow$

- Eine chirurgische Maßnahme kann als ultima ratio nach Scheitern wiederholter multimodaler konservativer Therapien bei extrem adipösen Jugendlichen mit erheblicher Komorbidität erwogen werden. $\Leftrightarrow$ 
- Können die als Kontraindikationen genannten Erkrankungen und Zustände erfolgreich behandelt werden oder können psychopathologische Zustände in einen stabilen Zustand überführt werden, sollte eine Reevaluation erfolgen. $\Uparrow$

\section{Kommentar}

Das US-amerikanische National Institute of Health (NIH) empfahl 1991 bariatrische Operationen bei einem BMI über $40 \mathrm{~kg} / \mathrm{m}^{2}$ oder über $35 \mathrm{~kg} / \mathrm{m}^{2}$ mit assoziierter Komorbidität. Diese Empfehlungen sollten die Entwicklung der Adipositaschirurgie in einem engen Indikationsbereich ermöglichen und dienten seither als Grundlage für Empfehlungen anderer Fachgesellschaften. Folgerichtig ist Evidenz nur innerhalb dieses a priori festgelegten Indikationsspektrums mit ausreichender Stärke generiert worden.

Für eine bariatrische Operation wird traditionell ein «Versagen» der konservativen Therapie zur Bedingung gemacht. In der Leitlinie wird der Begriff «Versagen» durch «Erschöpfung» ersetzt. Die konservativen Behandlungsmöglichkeiten gelten als erschöpft, wenn durch eine multimodale konservative Therapie das Ziel von 10-20\% Körpergewichtsverlust bei einem BMI von $35-40 \mathrm{~kg} / \mathrm{m}^{2}$ und von $10-30 \%$ Körpergewichtsverlust bei einem BMI $>40 \mathrm{~kg} / \mathrm{m}^{2}$ innerhalb von 6-12 Monaten nicht erreicht und gehalten werden kann. Zur konservativen Therapie gehören folgende Module:

- Ernährung: energiereduzierte Mischkost und eine weitere ernährungsmedizinische Maßnahme (z.B. Formula-Diät, weitere Form einer energiereduzierten Mischkost);

- Bewegung: Durchführung einer Ausdauer- und/oder Kraftausdauersportart mit mindestens $2 \mathrm{~h}$ Umfang pro Woche, falls keine Barrieren bestehen (z.B. Gonarthrose für Gehsportarten oder Scham beim Schwimmen);

- Psychotherapie: Durchführung einer ambulanten oder stationären Psychotherapie (Verhaltenstherapie oder Tiefenpsychologie), falls eine Essstörung («binge eating», «night eating») oder eine Psychopathologie (z.B. Depression, Ängstlichkeit) vorliegt;

- Patientengruppe: Behandlungen zum Lebensstil sollten nach Möglichkeit in der Gruppe durchgeführt werden.

Neu aufgenommen hat die Leitlinie die «primäre» Operationsindikation bei geringer Erfolgsaussicht der konservativen Therapie. Diese Situation trifft dann zu, wenn Art und/oder Schwere der Krankheit bzw. die psychosozialen Gegebenheiten eine rasche Gewichtsabnahme erforderlich machen oder wenn die konservative Therapie keinerlei Aussicht auf Erfolg hat. Die Indikation ist dann durch einen in der Adipositastherapie qualifizierten Arzt zusammen mit einem bariatrischen Chirurgen zu stellen.

Die Leitlinie nimmt den Diabetes mellitus Typ 2 als Indikationskriterium beim BMI $<35 \mathrm{~kg} / \mathrm{m}^{2}$ auf, was im Schrifttum als metabolische Chirurgie bezeichnet wird. Die streng selektionierten Patienten sollten dabei in Studien eingeschlossen werden (z.B. das prospektive Register der chirurgischen Fach- gesellschaft). Die Ausweitung des BMI-basierten Indikationsspektrums beruht auf den hohen Remissionsraten des Diabetes mellitus Typ 2 nach bariatrischen Eingriffen. In einer randomisierten Studie verglichen Dixon et al. [20] im Jahr 2008 (EL 1b) die Magenbandimplantation mit einer konservativen Therapie (Ermutigung zur Lifestyle-Änderung plus, wenn gewünscht, hypokalorische Ernährung) bei diabetischen Patienten mit einem BMI zwischen 30 und $40 \mathrm{~kg} / \mathrm{m}^{2}$. Nach 2 Jahren wurden mit dem Magenband 62,5\% des Übergewichts reduziert gegenüber 4,3\% mit konservativer Therapie. Die Rückbildungsrate des Diabetes (Nüchternblutzucker < 126 mg/dl; HbA1c < 6,2\% ohne Medikation) betrug $73 \%$ nach Magenband gegenüber $13 \%$ unter konservativer Therapie. In der Metaanalyse von Buchwald et al. [4] (EL1a) von 2009 lag die Remissionsrate des Diabetes mellitus Typ 2 im Mittel bei 76,8\%: 38\% nach Magenband, $84 \%$ nach Magen-Bypass und 98\% nach biliopankreatischer Diversion (BPD) mit oder ohne Duodenal-Switch (DS).

Dieser metabolische Effekt ist bemerkenswert, weil er bei den kombiniert restriktiv-malabsorptiven Operationen noch vor einem signifikanten Gewichtsverlust eintritt (EL 4) [21]. Die Medikamentendosis kann in der Regel frühpostoperativ verringert und bei vielen Patienten innerhalb von 3 Monaten abgesetzt werden. Dieser Effekt bleibt über viele Jahre stabil (EL 2b) [5]. Zudem konnten bariatrische Eingriffe die spätere Entstehung von Diabetes mellitus Typ 2 in einer longitudinalen Vergleichsanalyse [22] verhindern (EL 1b).

In der aktuellen Leitlinie gibt es keine Altersbeschränkungen mehr. Bei Jugendlichen soll die Operationsentscheidung mit höchster Sorgfalt gestellt werden; hier wurde in Konkordanz mit anderen Leitlinien der Begriff «Versagen» beibehalten. Die Liste an Kontraindikationen wird bis auf wenige therapierefraktäre oder konsumierende Erkrankungen, wie z.B. ein Leberschaden mit portaler Hypertension, kongenitale Fehlbildungen im oberen Gastrointestinaltrakt, unbehandelte oder unzureichend eingestellte psychische Erkrankungen oder Drogen- und Alkoholabusus, zusammengestrichen. Fehlende Compliance oder mangelnder Intellekt des Patienten zur Ernährungsumstellung und Nachsorge sowie eine aktuelle Schwangerschaft stehen einer Operation entgegen.

\section{Verfahrenswahl}

- Ein für alle Patienten pauschal zu empfehlendes Verfahren existiert nicht. $\Uparrow \Uparrow$

- Als effektive operative Verfahren zur Therapie der Adipositas sollten Magenband, Schlauchmagen, Roux-YMagen-Bypass oder BPD mit DS (BPD-DS) eingesetzt werden. $\Uparrow$

- Ferner können Ein-Anastomosen-Bypass, BPD oder vertikale Bandplastik eingesetzt werden. $\Leftrightarrow$

- Die Verfahrenswahl soll unter Berücksichtigung von BMI, Alter, Geschlecht, Komorbiditäten, Adhärenz und Beruf erfolgen. $\Uparrow \Uparrow$ 
- Der Patient soll über die gängigen Verfahren beraten werden. Hierzu gehören Magenband, Magen-Bypass, Schlauchmagen, BPD-DS sowie im Einzelfall Stufenkonzepte (z.B. Magenballon oder Schlauchmagen als erster Schritt). Der Patient ist über Operationsverfahren und mögliche Behandlungsalternativen, Therapieeffekte, Komplikationen einschließlich Sterblichkeit, Nachsorge inklusive möglicher lebenslanger Supplementation und plastische Folgeoperationen aufzuklären. $\Uparrow \Uparrow$

- Die Präferenz des aufgeklärten Patienten soll bei der Verfahrenswahl und bei Fehlen von Kontraindikationen berücksichtigt werden. $\Uparrow \Uparrow$

- Zweizeitige Konzepte (Stufenkonzepte) sind in der Lage, das perioperative Risiko zu senken, und sollten besonders bei Patienten mit Extremformen der Adipositas (BMI > $50 \mathrm{~kg} / \mathrm{m}^{2}$ ) und/oder erheblicher Komorbidität erwogen werden. $\Uparrow$

- Auch der Einsatz eines Magenballons kann im Einzelfall zur kurzfristigen Gewichtsreduktion erwogen werden. $\Leftrightarrow$

- Die Operationen sollen von Chirurgen mit Expertise und in Krankenhäusern mit institutioneller Erfahrung in der Adipositaschirurgie durchgeführt werden. $\Uparrow \Uparrow$

\section{Kommentar}

In Deutschland haben sich Magenband, Magen-Bypass und BPD-DS als gängige Verfahren durchgesetzt und werden deshalb in der Leitlinie als Standardverfahren bezeichnet. In drei umfangreichen Metaanalysen zeigten Buchwald et al. [2-4] (EL 1a), dass das Ausmaß des mittleren Gewichtsverlusts vom Magenband (47,5\%; 95\%-Konfidenzintervall (CI) 40,7$54,2)$ über den Magen-Bypass (61,6\%; 95\%-CI 56,7-66,5) bis zur BPD mit oder ohne DS anstieg (70,1\%; 95\%-CI 66,373,9) (136 Arbeiten mit 22094 Patienten [2]). Die postoperative 30-Tage-Letalität betrug nach restriktiven Verfahren 0,07\% (95\%-CI 0,02-0,12), 0,16\% (95\%-CI 0,09-0,23) nach dem Magen-Bypass und 1,11\% (95\%-CI, 0,00-2,70) nach malabsorptiven Verfahren (361 Arbeiten mit 85048 Patienten [3]). Die jüngste Metaanalyse [4] mit 621 Arbeiten und 135246 Patienten fand eine Besserung der diabetischen Stoffwechselsituation bei $87 \%$ und eine komplette Remission bei $78 \%$ der operierten Patienten. Die Analyse legt den Schluss nahe, dass invasivere Verfahren einen stärkeren Effekt haben als weniger invasive.

Der Schlauchmagen («sleeve gastrectomy») gilt nun ebenfalls als Standardoperation, auch wenn für diese recht neue Operationstechnik weder Langzeitdaten noch metaanalytische Vergleiche vorliegen. Der Schlauchmagen erfährt gegenwärtig nicht nur als vorbereitender, sondern auch als eigenständiger Eingriff weltweit eine rasante Verbreitung, denn auch bei Patienten mit niedrigerem BMI (30-40 kg/m²) sind restriktive Verfahren effektiv und der konservativen Therapie deutlich überlegen (EL 1b) [20]. Allmählich beginnt man zu verstehen, dass der Magenschlauch kein rein restriktives Verfahren ist, sondern neurohumorale Veränderungen induziert, die dazu beitragen, dass der postoperative Gewichtsverlust höher ist als der nach Magenbandimplantation (EL 1b) [23] und eher dem nach Magen-Bypass gleicht.

Die 30-Tage-Letalität beträgt nach Magenband 0,1\%, nach Magen-Bypass 0,2\% und nach BPD-DS 0,7\% (EL 1a) [2]. Der Schlauchmagen zeigt eine mittlere Letalität von $0,3 \%$ (EL 4) [15]. Die Rate an schweren Komplikationen, wie Anastomoseninsuffizienzen, Nachblutungen und Lungenembolien, beträgt weniger als 5\%. Morbidität und Letalität sind nicht nur abhängig vom Operationsverfahren, sondern auch von der Erfahrung des Chirurgen (EL 4) [16]. Deshalb sollten die Operationen von Chirurgen mit Expertise und in Krankenhäusern mit institutioneller Erfahrung in der Adipositaschirurgie durchgeführt werden.

Chirurgische Spätkomplikationen sind vor allem innere Hernien und Magenbandkomplikationen (EL 2b) [17] wie Slippage (Gleiten) und Migrationen (EL 2b) [17]. Portkomplikationen (Rotation, Diskonnektion, Penetration, Infektionen) treten in bis zu 5\% der Fälle auf und können häufig in Lokalanästhesie korrigiert werden.

Zweizeitige Konzepte (Stufenkonzepte) sind in der Lage, das perioperative Risiko zu senken, und sollten besonders bei Patienten mit Extremformen der Adipositas (BMI $>50 \mathrm{~kg} / \mathrm{m}^{2}$ ) und/oder erheblicher Komorbidität erwogen werden. So kann die Schlauchmagenbildung als erster Schritt vor einem späteren Magen-Bypass oder DS eingesetzt werden. Der endoskopisch platzierte Magenballon kann im Einzelfall zur kurzfristigen vorbereitenden Gewichtsreduktion erwogen werden.

Einen operativen «Goldstandard» gibt es nicht. Auch ist derzeit keine standardisierte, auf objektiven Parametern basierende Verfahrenswahl möglich. Die Verfahrenswahl soll deshalb unter Berücksichtigung von BMI, Alter, Geschlecht, Komorbiditäten, Adhärenz und psychosozialen Gegebenheiten individuell erfolgen.

\section{Technische Aspekte und Komplikationen}

- Magenballon: Bei flüssigkeitsbefüllten Ballons soll Methylenblau zugesetzt werden, um frühzeitig einen spontanen Ballonkollaps zu bemerken. $\Uparrow \Uparrow$

- Bei gastralen Voroperationen soll aufgrund der erhöhten Perforationsgefahr auf die Implantation eines Magenballons verzichtet werden. $\Uparrow \Uparrow$

- Magenband: Bei der Anlage eines Magenbands soll die Positionierung durch die Pars flaccida des Omentum minus erfolgen, um so die Rate an Banddislokationen auf ein Minimum zu begrenzen. $\Uparrow \Uparrow$

- Die Anlage soll grundsätzlich laparoskopisch erfolgen. $\Uparrow \Uparrow$

- Roux-Y-Magen-Bypass: Die Anlage eines MagenBypasses soll laparoskopisch erfolgen. $\Uparrow \Uparrow$ 
- Der Magenpouch sollte klein sein. $\Uparrow$

- Die Länge des alimentären Schenkels sollte ungefähr $150 \mathrm{~cm}$ betragen, um einen adäquaten Gewichtsverlust bei nur geringen metabolischen Komplikationen zu erreichen. $\Uparrow$

- Der alimentäre Schenkel sollte antekolisch-antegastrisch positioniert werden. $\Uparrow$

- BPD-DS: Die BPD mit DS sollte laparoskopisch durchgeführt werden. $\Uparrow$

- Die Länge des gemeinsamen Schenkels sollte ungefähr $100 \mathrm{~cm}$ betragen. $\Uparrow$

- Schlauchmagen: Der Eingriff soll grundsätzlich laparoskopisch erfolgen. $\Uparrow \Uparrow$

- Zur Bildung des Magenschlauchs sollte eine Kalibrierung erfolgen. $\Uparrow$

- Simultane Cholezystektomie: Eine simultane Cholezystektomie wird bei Patienten mit präoperativer symptomatischer Cholelithiasis empfohlen. $\Uparrow$

- Bei asymptomatischer Cholezystolithiasis kann eine prophylaktische Cholezystektomie in Erwägung gezogen werden. $\Leftrightarrow$

- Narbenhernie: Die elektive operative Behandlung einer postoperativen Narbenhernie sollte bis zum Erreichen eines stabilen Gewichts und verbesserten Ernährungszustands aufgeschoben werden. $\Uparrow$

- Postbariatrische plastische Chirurgie: Die Veränderungen des äußeren Erscheinungsbilds und der damit verbundenen medizinischen und psychosozialen Folgen müssen schon vor der bariatrischen Operation mit dem Patienten besprochen werden. $\Uparrow \Uparrow$

- Die plastisch-chirurgischen Korrekturen nach erfolgreicher Gewichtsreduktion sollten ein integraler Bestandteil des Gesamtbehandlungskonzepts sein. $\Uparrow$

\section{Kommentar}

Im umfangreichen Begleittext der Originalversion wird die Literatur zu den verschiedenen operativ-technischen Varianten diskutiert. Die evidenzbasierten Standards haben mittlerweile breiten Eingang in die chirurgischen Operationslehren gefunden und sollen hier nicht wiederholt werden. Die Kernaussage ist die, dass die bariatrische Chirurgie eine laparoskopische ist - kein anderes Gebiet der Viszeralchirurgie ist so unumstritten laparoskopisch geworden. Dies gilt auch für Revisionen aufgrund von Komplikationen oder Wiederholungseingriffen. Damit gibt die bariatrische Chirurgie Impulse an das ganze Fach ab. Weitere praxisrelevante Empfehlungen sind die der simultanen Cholezystektomie bei symptomatischen Gallensteinen und die aufgeschobene Reparatur von Narbenhernien nach dem Ende des Gewichtsverlusts. In der Leitlinie wird die postbariatrische plastische Körperkorrektur in das Gesamtbehandlungskonzept der Adipositas integriert. Damit weist die Leitlinie auf die drastischen Veränderungen des äußeren Erscheinungsbilds und die medizinische Notwendigkeit zu Korrektureingriffen hin.
Nachsorge

- Patienten nach adipositaschirurgischen Eingriffen bedürfen einer regelmäßigen Nachsorge durch einen in der Adipositastherapie erfahrenen Arzt und eine Ernährungsfachkraft. $\Uparrow \Uparrow$

- Bariatrisch operierten Patienten sollte im ersten postoperativen Jahr eine engmaschige Nachsorge und ärztliche Betreuung angeboten werden. $\Uparrow$

- Die Supplementierung von Vitaminen und Mineralien soll nach malabsorptiven und Kombinationseingriffen routinemäßig erfolgen, ebenso bei rein restriktiven Verfahren bei sehr starkem Gewichtsverlust und bei wiederholtem Erbrechen. $\Uparrow \Uparrow$

- Mittels Laborkontrolle sollen Mangelerscheinungen rechtzeitig erkannt werden. $\Uparrow \Uparrow$

- Die postoperative Behandlung durch einen Psychologen/Psychosomatiker/Psychiater kann bei präoperativ manifester psychischer Störung und bei postoperativem Auftreten von Essanfällen («loss of control eating") oder von anderen psychischen Störungen empfohlen werden. $\Leftrightarrow$

- Die Teilnahme an Selbsthilfegruppen kann empfohlen werden. $\Leftrightarrow$

- Bei Patientinnen im gebärfähigen Alter sollte eine Empfängnisverhütung während des raschen $\mathrm{Ge}$ wichtsverlusts durchgeführt werden. $\Uparrow$

- Dosisanpassungen von Medikamenten müssen gegebenenfalls durchgeführt werden. $\Uparrow \Uparrow$

\section{Kommentar}

Die Leitlinie sieht die Adipositas als eine chronische Erkrankung, die auch nach einer bariatrischen Operation einer kompetenten ärztlichen und ernährungsmedizinischen Betreuung bedarf. Allerdings gibt es kein evidenzbasiertes und allgemein anerkanntes Nachsorgeschema. Die Nachsorgeintervalle richten sich nach der durchgeführten Operation, der Dynamik des Gewichtsverlusts und nach auftretenden Problemen und Komplikationen. Am häufigsten treten sie im ersten postoperativen Jahr auf, weswegen in diesem Zeitraum die Nachsorgetermine engmaschiger vergeben werden müssen. Gängige Praxis sind im ersten postoperativen Jahr dreimonatliche, im zweiten Jahr halbjährliche und bei Gewichtsstabilität jährliche Nachuntersuchungen.

In der Phase des raschen Gewichtsverlusts ist eine proteinreiche Kost mit ausreichender Supplementation von Spurenelementen (Eisen), Mineralien (Calcium) und Vitaminen (Vitamin B12, Vitamin D) notwendig. Die Supplementation ist nach Kombinationseingriffen wie dem Magen-Bypass lebenslang erforderlich. Aus ernährungsmedizinischer Sicht wird eine langfristige Umstellung empfohlen, die allgemeinen Standards für eine ausgewogene Kost entspricht.

In der Nachsorge sollen rechtzeitig Spätkomplikationen und Mangelzustände mittels klinischer und Laboruntersu- 
chungen erkannt werden. Letztere beinhalten: Blutbild, Nierenretentionswerte, Elektrolyte, Zink, Eisen, Eiweiß, Albumin, Cholesterin, HDL, LDL, Nüchternblutzucker, HbA1c, Parathormon, Folsäure, Vitamin B12 und Vitamin D (EL4) [24]. Durch regelmäßige postoperative Nachsorge kann der Gewichtsverlust größer sein als bei Vernachlässigung (EL 4) $[25,26]$. Beim Magenband wird die Justierung in mehreren Schritten vorgenommen.

Die Operation ist keine Lösung seelischer Probleme. Eine fachkompetente Behandlung sollte beim Neu- oder Wiederauftreten psychischer Störungen eingeleitet werden. Selbsthilfegruppen können einen positiven Effekt im postoperativen Verlauf ausüben und den Gewichtsverlust fördern (EL 4) [27, 28].

\section{Diskussion}

Die Adipositaschirurgie entwickelt sich in Deutschland langsamer als in vielen anderen Ländern. Dies steht im Gegensatz zur gesicherten Erkenntnis, dass die bariatrische Chirurgie die effektivste Form der Adipositastherapie ist. Mit ihr kann auch bei Patienten mit hohem BMI $\geq 40 \mathrm{~kg} / \mathrm{m}^{2}$ eine ausreichende und dauerhafte Gewichtsabnahme erreicht werden. Die Deutsche Gesellschaft für Chirurgie hat diese wissenschaftlich fundierte State-of-the-Art-Übersicht mit dem Ziel initiiert, interdisziplinär konsentierte evidenzbasierte anstelle von Bestclinical-practice-Empfehlungen zu formulieren, um die bariatrische Chirurgie im multimodalen Behandlungskonzept der Adipositas zu etablieren. Gleichzeitig wollte die Gesellschaft ein System zur Qualitätssicherung implementieren, das neben einem nationalen Register, das der Versorgungsforschung und dem Benchmarking dient, auch wissenschaftlich fundierte klinisch relevante Empfehlungen beinhaltet.

Die evidenzbasierten Empfehlungen der S3-Leitlinie dienen als Entscheidungshilfen. Vorbereitung und Nachsorge sollen interdisziplinär durchgeführt werden. Eine Operation ist nach Erschöpfung oder Aussichtslosigkeit der konservativen Therapie indiziert. Das traditionelle Indikationsspektrum $\left(\mathrm{BMI}>40 \mathrm{~kg} / \mathrm{m}^{2}\right.$ oder $>35 \mathrm{~kg} / \mathrm{m}^{2}$ mit Komorbiditäten) wird durch den Wegfall der Altersgrenzen und Kontraindikationen erweitert. Die metabolische Chirurgie des Diabetes mellitus Typ 2 wird innerhalb von Studien als Option betrachtet. Die Laparoskopie ist Zugangsweg der Wahl für Primäreingriffe. Magenband, Magen-Bypass und BPD-DS sind Standardeingriffe, wozu nun auch der Schlauchmagen insbesondere im Rahmen der Mehrschritttherapie bei extremer Adipositas gezählt wird. Die Verfahrenswahl richtet sich nach dem Ausgangsgewicht und einer Reihe weiterer individueller Kriterien.

Da die Ergebnisse der Konsensus-Konferenz von einer interdisziplinär zusammengesetzten Kommission entwickelt wurden, ist zu hoffen, dass die Leitlinie breite Akzeptanz bei Ärzten und Kostenträgern findet. Die Stärke dieser Leitlinie ist der breite Konsens der Chirurgen mit den nichtchirurgischen Kommissionsmitgliedern. Empfehlungen können in einer S3-Leitlinie nur bei vorliegender Evidenz ausgesprochen werden, sodass das Fehlen von Empfehlungen zu einem bestimmten Thema den ärztlichen Entscheidungsspielraum öffnet und die Notwendigkeit für weitere wissenschaftliche Studien aufzeigt. Dies betrifft insbesondere vergleichende Studien zur Verfahrenswahl und zur strukturierten Nachsorge. Die Leitlinie hat eine Gültigkeit von 3 Jahren.

\section{Mitglieder der Kommission}

Deutsche Gesellschaft für Allgemein- und Visceralchirurgie (DGAV): Prof. Dr. Dr. h.c. N. Runkel, Villingen-Schwenningen; PD Dr. M. Colombo-Benkmann, Münster; PD Dr. T.P. Hüttl, München; PD Dr. O. Mann, Hamburg; Dr. Ricarda Flade-Kuthe, Hannover; Prof. Dr. E. Shang, Mannheim; Dr. M. Susewind, Berlin; Dr. H. Tigges, Konstanz; PD Dr. S. Wolff, Magdeburg; Dr. R. Wunder, Hannover. Deutsche Adipositasgesellschaft (DAG): Prof. Dr. A. Wirth, Bad Rothenfelde; Dr. K. Winckler, Frankfurt/M. Deutsche Gesellschaft für Ernährungsmedizin: Prof. Dr. A. Weimann, Leipzig. Deutsche Gesellschaft für Psychosomatische Medizin und Ärztliche Psychotherapie: Prof. Dr. Martina de Zwaan, Erlangen. Methodiker: Prof. Dr. S. Sauerland, Köln.

\section{Disclosure Statement}

Keine Mitglieder der Kommission haben irgendwelche direkten Mittel der Industrie erhalten. Die gesamte Leitlinienerstellung war frei von $\mathrm{Zu}$ wendungen Dritter.

\section{Literatur}

1 Fontaine KR, Redden DT, Wang C, Westfall AO, Allison DB: Years of life lost due to obesity. JAMA 2003;289:187-193.

2 Buchwald H, Avidor Y, Braunwald E, Jensen MD, Pories W, Fahrbach K, Schoelles K: Bariatric surgery: a systematic review and metaanalysis. JAMA 2004:292:1724-1737.

3 Buchwald H, Estok R, Fahrbach K, Banel D, Sledge I: Trends in mortality in bariatric surgery: a systematic review and meta-analysis. Surgery 2007 ; 142:621-632.
Buchwald H, Estok R, Fahrbach K, Banel D, Jensen MD, Pories WJ, Bantle JP, Sledge I: Weight and type 2 diabetes after bariatric surgery: systematic review and meta-analysis. Am J Med 2009;122: 248-256.

5 Sjöström L, Lindroos AK, Peltonen M, Torgerson J, Bouchard C, Carlsson B, Dahlgren S, Larsson B, Narbro K, Sjöström CD, Sullivan M, Wedel H; Swedish Obese Subjects Study Scientific Group: Lifestyle, diabetes, and cardiovascular risk factors 10 years after bariatric surgery. N Engl J Med 2004; 351:2683-2693.
6 Sjöström L, Narbro K, Sjöström CD et al: Effects of bariatric surgery on mortality in Swedish obese subjects. N Engl J Med 2007;357:741-752.

7 Adams TD, Gress RE, Smith SC, Halverson RC, Simper SC, Rosamond WD, Lamonte MJ, Stroup AM, Hunt SC: Long-term mortality after gastric bypass surgery. N Engl J Med 2007;357:753-761. 
8 Sauerland S, Angrisani L, Belachew M, Chevallier JM, Favretti F, Finer N, Fingerhut A, Garcia Caballero M, Guisado Macias JA, Mittermair R, Morino M, Msika S, Rubino F, Tacchino R, Weiner R, Neugebauer EA; European Association for Endoscopic Surgery: Obesity surgery: evidence-based guidelines of the European Association for Endoscopic Surgery (EAES). Surg Endosc 2005;19:200-221.

$\checkmark$ Melissas J, Christodoulakis M, Schoretsanitis G, Sanidas E, Ganotakis E, Michaloudis D, Tsiftsis DD: Obesity-associated disorders before and after weight reduction by vertical banded gastroplasty in morbidly vs superobese individuals. Obes Surg 2001;11:475-481.

10 Catheline JM, Bihan H, Le Quang T, Sadoun D, Charniot JC, Onnen I, Fournier JL, Bénichou J, Cohen R: Preoperative cardiac and pulmonary assessment in bariatric surgery. Obes Surg 2008;18:271-277.

11 Hallowell PT, Stellato TA, Schuster M, Graf K, Robinson A, Crouse C, Jasper JJ: Potentially lifethreatening sleep apnea is unrecognized without aggressive evaluation. Am J Surg 2007;193:364-367.

- 12 Heo M, Pietrobelli A, Fontaine KR, Sirey JA, Faith MS: Depressive mood and obesity in US adults: comparison and moderation by sex, age, and race. Int J Obes 2006;30:513-519.

13 Simon GE, Von Korff M, Saunders K, Miglioretti DL, Crane PK, van Belle G, Kessler RC: Association between obesity and psychiatric disorders in the US adult population. Arch Gen Psychiatry 2006;63:824-830.
14 de Zwaan M, Wolf AM, Herpertz S: Psychosomatische Aspekte der Adipositaschirurgie. Dtsch Ärztebl 2007;104:2577-2583.

15 Ashton D, Favretti F, Segato G: Preop psychological testing - another form of prejustice. Obes Surg 2008;18:1330-1337.

16 Kim TH, Daud A, Ude AO, DiGiorgi M, OliveroRivera L, Schrope B, Davis D, Inabnet WB, Bessler M: Early U.S. outcomes of laparoscopic gastric bypass versus laparoscopic adjustable silicone gastric banding for morbid obesity. Surg Endosc 2006;20:202-209.

17 Marcus MD, Kalarchian MA, Courcoulas AP: Psychiatric evaluation and follow-up of bariatric surgery patients. Am J Psychiatry 2009;166:285-291.

18 Alami RS, Morton JM, Schuster R, Lie J, Sanchez BR, Peters A, Curet MJ: Is there a benefit to preoperative weight loss in gastric bypass patients? A prospective randomized trial. Surg Obes Relat Dis 2007;3:141-145.

19 Colles SL, Dixon JB, Marks P, Strauss BJ, O'Brien PE: Preoperative weight loss with a very lowenergy diet: quantitation of changes in liver and abdominal fat by serial imaging. Am J Clin Nutr 2006;84:304-311.

20 Dixon JB, O'Brien PE, Playfair J, Chapman L, Schachter LM, Skinner S, Proietto J, Bailey M, Anderson M: Adjustable gastric banding and conventional therapy for type 2 diabetes: a randomized controlled trial. JAMA 2008;299:316-323.

21 Rubino F, Gagner M: Potential of surgery for curing type 2 diabetes mellitus. Ann Surg 2002;236: $554-559$
22 O'Brien PE, Dixon JB, Laurie C, Skinner S, Proietto J, McNeil J, Strauss B, Marks S, Schachter L, Chapman L, Anderson M: Treatment of mild to moderate obesity with laparoscopic adjustable gastric banding or an intensive medical program: a randomized trial. Ann Intern Med 2006;144:625-633.

23 Himpens J, Dapri G, Cadiere GB: A prospective randomized study between laparoscopic gastric banding and laparoscopic isolated sleeve gastrectomy: results after 1 and 3 years. Obes Surg 2006; 16:1450-1456.

24 Ziegler O, Sirveaux MA, Brunaud L, Reibel N, Quilliot D: Medical follow up after bariatric surgery: nutritional and drug issues. General recommendations for the prevention and treatment of nutritional deficiencies. Diabetes Met 2009;35 (6 pt 2):544-557.

25 Gould JC, Beverstein G, Reinhardt S, Garren MJ Impact of routine and long-term follow-up on weight loss after laparoscopic gastric bypass. Surg Obes Relat Dis 2007;3:627-630.

26 Harper J, Madan AK, Ternovits CA, Tichansky DS: What happens to patients who do not follow-up after bariatric surgery? Am Surg 2007:73:181-184.

27 Orth WS, Madan AK, Taddeucci RJ, Coday M, Tichansky DS: Support group meeting attendance is associated with better weight loss. Obes Surg 2008;18:391-394.

28 Song Z, Reinhardt K, Buzdon M, Liao P: Association between support group attendance and weight loss after Roux-en-Y gastric bypass. Surg Obes Relat Dis 2008;4:100-103. 